\title{
Briefly about mesenchymal stem cells - one of the main players in bone tissue engineering
}

\begin{abstract}
Mesenchymal stem cells (MSCs) are multipotent stem cells with high self-renewal capacity, ability to differentiate into osteoblasts, adipocytes and chondroblasts, immunomodulating properties and lack of teratogenic potential. These cells have been recognized to be suitable for the needs of regenerative medicine and bone tissue engineering. The presented mini-review summarizes information about biological characteristics (advantages and challenges) of MSCs of various tissue origin.
\end{abstract}

Keywords: mesenchymal stem cells, bone diseases, bone tissue engineering, regenerative medicine

\author{
Volume 3 Issue I - 2018 \\ Radostina Alexandrova, ' Tanya Zhivkova,' \\ Lora Dyakova, ${ }^{2}$ Boyka Andonova-Lilova,' \\ Desislav Dinev,' Abedulkadir Abudalleh,' \\ Nabanita Saha, ${ }^{3}$ Virginija Jankauskaite, ${ }^{4}$ Orlin \\ Alexandrov ${ }^{5}$ \\ 'Department of Pathology, Institute of Experimental \\ Morphology, Pathology and Anthropology with Museum, \\ Bulgaria \\ ${ }^{2}$ Department of Synaptic Signaling and Communications, \\ Institute of Neurobiology, Bulgaria \\ ${ }^{3}$ Thomas Bata University in Zlin, Czech Republic \\ ${ }^{4}$ Department of Design and Technologies, Kaunas University of \\ Technology, Lithuania \\ ${ }^{5}$ Health Service, Bulgaria
}

Correspondence: Radostina Alexandrova, Department of Pathology, Institute of Experimental Morphology, Pathology and Anthropology with Museum, Bulgarian Academy of Sciences, Bulgaria, Fax +3592-87 I0-107, Tel +3592-979-3678, Email rialexandrova@hotmail.com

Received: January 29, 2018 | Published: February 14, 2018

\section{Bone diseases}

Bone joint pathologies caused by several diseases, congenital defects, infections and trauma significantly decrease the quality of human's life, may be life-limiting and are recognized to be among the major health and social challenges of our time., ${ }^{1,2}$ Bone has a remarkable regenerative potential - fracture healing is a complex process that follows specific regenerative patterns involving several molecular, genetic, biochemical and cellular mechanisms. When the size of the lost bone is greater than the healing capacity of osteogenic tissues there is a need of bone implants. Nowadays, autologous (the gold standard for repairing bone defects) and allogeneic bone grafts are still widely used in clinical practice for repairing bone defects but their application has been connected with various challenges including limited availability, additional surgical trauma and postoperative complications (in the case of autografts), foreign body immune reactions, risk of infections (for example transmission of Hepatitis C virus, Human immunodeficiency virus), poor degree of cellularity, less revascularization and a higher resorption rate (in the case of allografts). ${ }^{3,4}$ That is why there is an urgent need for design of suitable substitute with improved incorporation, osteogenicity, osteoconductivity, and osteoinductivity that can mimic to the highest degree the biological, physical and mechanical characteristics of the normal autogenous bone.

\section{Bone tissue engineering}

Bone tissue engineering (BTE) is a strategy combining the knowledge and principles of orthopedics, bioengineering, molecular/ cellular biology, cell transplantation, and materials' science to construct substitutes that can restore and maintain normal functions of injured and diseased bone. This innovative approach involves the use of stem cells that are seeded into 3D biocompatible scaffolds and induced by appropriate stimulation (growth factors, cytokines, etc) to generate genuine new bone. ${ }^{2,4,5}$

\section{Stem cells}

Stem cells (SCs) are unspecialized types of cells with the ability to proliferate, self-renew and differentiate under certain physiologic conditions and signaling. The initial concept of stem cells appeared more than one century ago. Although the major advances in clarifying the biology / behavior of SCs that occurred over the last three decades, our knowledge about their nature and ability to explore therapeutic potential are still insufficient. ${ }^{6,7}$ As compared to embryonic stem cells (ESCs) the adult (or somatic) stem cells are rare, quiescent cells with a more limited self-renewal and differentiation. At the same time, adult stem cells have two very important advantages: they circumvent ethical issues and show less tumorigenicity. Thousands of clinical trials using stem cells are currently in progress. ${ }^{8}$

\section{Mesenchymal stem cells}

Mesenchymal stem cells (MSCs - multipotent stem cells with fibroblast-like morphology) are of mesodermal origin, except for the facial bones, which arise from neural crest. ${ }^{9}$ MSCs can be isolated from several adult tissues (bone marrow, adipose tissue, peripheral blood, etc) as well as from birth associated /neonatal tissues (BA/ NTs) (placenta, amnion, umbilical cord and cord blood) and are found in both embryonic and adult tissues in humans. The minimal set of criteria for identification/characterization of MSCs have been 
proposed by the International Society for Cellular Therapy (ISCT): First, MSCs must be plastic-adherent when maintained in standard culture conditions. Second, MSCs have to be positive for the presence of CD105, CD73 and CD90 surface molecules (these markers should be expressed by more than $95 \%$ of the anticipated MSCs population) with no expression (less than $2 \%$ of the cells) of CD45, CD34, CD14 or CD11b, CD79alpha or CD19 and HLA-DR. Third, MSC must differentiate (at least) to osteoblasts, adipocytes and chondroblasts under standard in vitro inductive conditions. ${ }^{10}$ The yield and quality (proliferation activity, differentiation potential) of MSCs depend on tissue source, donor age (decrease with increasing donor age) and disease stage/health status. The decreased quantity and quality of MSCs due to the advance age of the patient may limit the application of autologous MSCs in clinical practice. MSCs have been recognized to be suitable for BTE because of their relatively easy access and high availability, capacity for extensive self-renewal or expansion to generate sufficient amount, the ability to differentiate into osteoblasts and chondroblasts, immunomodulating properties, lack of teratogenic potential. ${ }^{2,11,12}$

\section{Bone marrow mesenchymal stem cells}

The first multipotent mesenchymal stem cells identified were the bone marrow stromal/stem cells (BM-MSCs), described as colonyforming unit-fibroblasts in vitro. They are still the most frequently investigated cell type and are often designated as the gold standard. ${ }^{13}$ BM-MSCs are very attractive for the needs of regenerative medicine because of their high differentiation potential. The main obstacles associated with their application in this field are:

i) MSCs are a rare population in bone marrow $(0.001-0.01 \%$ of the total nucleated cells)

ii) harvesting bone marrow is an invasive procedure carrying a potential risk for infection. BM-MSCs are limited to a growth potential of 30-50 population doubling following ex vivo expansion. ${ }^{11,14,15}$

\section{Adipose stem cells}

Adipose stem cells (ASCs) possess some specific features that makes them particularly suitable for the needs of BTE. Fpr example, as compared to BMSCs, ASCs have the following advantages:

i) they can be harvested in large amounts and readily expanded diminishing in this way the complications that accompany cultivation in laboratory conditions.

ii) more pronounced pro-angiogenic activity.

iii) higher genetic stability in long-term cultures. ${ }^{16,17}$

ASCs produce biologically active molecules that play important roles in bone regeneration/remodelling (bone morphogenic proteins-2 and -4 ; receptor activator of nuclear factor k-B ligand - RANKL; macrophage colony-stimulating factor; fibronectin; type I collagen); wound healing (fibroblast growth factor-2; keratinocyte growth factor; insulin-like growth factor-1) and angiogenesis (vascular endothelial growth factor-VEGF, insulin-like growth factor-1; matrix metalloproteinase enzymes MMP-3 and MMP-9; interleukin-8). The plasma membrane-derived vesicles (MVs) secreted by ASCs allow them to influence the behaviour of targeted cells situated not only in the neighborhood (paracrine effects) but also at distant locations throughout the body stimulating their proliferation and differentiation as well as the regenerative and reparative processes. These MVs contain and deliver various growth factors, cytokines, RNAs and micro RNAs. Last, but not the least, ASCs can survive under conditions of hypoxia, making them extremely valuable for bone tissue engineering applications, where the limited blood flow to the implant can be a challenge. ${ }^{18,19}$

\section{Birth associated/neonatal tissues}

BA/NTs have some specific advantages as a source for MSCs isolation such as:

i) as medical waste tissues some of them (placenta, umbilical cord) are easily accessible thus avoiding invasive procedures and ethical problems;

ii) The presence of various ESCs, MSCs, endothelial progenitor cells and hematopoietic stem cells $\left(\mathrm{CD} 34^{+}, \mathrm{CD} 133^{+}\right)$.

iii) There are data suggesting that MSCs derived from BA/NTs exhibit improved proliferative capacity, life span and differentiation potential as compared to BM-MSCs. ${ }^{20-23}$

Placental tissue can be of fetal or maternal origin which has to be taken into account during isolation and characterization of MSCs. Maternal MSCs are generally derived from the placental tissue, whereas fetal MSCs are usually obtained from the umbilical cord, amniotic membrane, and amniotic fluid. ${ }^{24}$ Cord blood (CB) has been reported to be the most reliable and attractive source for fetal MSCs because of at least two reasons:

i) No ethical and regulatory limitations associated with ESCs.

ii) CB-MSCs have been suggested to be safer for clinical application as compared to ESCs (no teratoma produced by CB-MSCs have been reported). ${ }^{2,25}$

The availability of private and public $\mathrm{CB}$ banks facilitates the proper storage and possible therapeutic application of CB-MSCs. Human endometrium is a highly dynamic tissue that undergoes more than 400 menstrual cycles of proliferation, differentiation and shedding during a woman's lifetime. It has recently been identified as an accessible source of MSCs that can be easily harvested via endometrial biopsy tissue or from menstrual blood. The biological characteristics of endometrial MSCs have been reported to be similar to those of BM-MSCs and ASCs. ${ }^{24,26}$

\section{Dental/oral stem cells}

Mesenchymal stem cells can be isolated from several dental locations (pulp, exfoliated primary deciduous teeth, apical papilla, dental follicule, periodontal ligament) as well as from some other oral tissues (gingiva, oral mucosa, craniofacial bone). ${ }^{27}$ Dental stem cells (DSCs) are derived from neural crest post natal stem cell populations with MSC-like characteristics. Compared to BM-MSCs, the DMSCs appear to be more related to odontogenic than osteogenic development. At the same time, it has been reported that human dental pulp stem cells (DPSCs) can co-differentiate into osteoblasts and endotheliocytes and have been suggested to be beneficial for solving one of the major problems in tissue engineering regarding angiogenesis. ${ }^{28}$ Although widely studied for their promising clinical potential, there are some obstacles in using DSCs for cell therapy, such as their limited tissue sources and the requirement for tooth extraction. Fox example, a very low number of DPSCs can be obtained because of the small size of the pulp (especially in the case of exfoliated deciduous teeth). 
Obtaining a large amount of cells sufficient for clinical application requires long term cultivation in laboratory conditions that might reduce the differentiation potency of the cells and could result in undesired genetic and/or epigenetic changes. ${ }^{29}$ There are data revealing that DPSCs can be cultured for 6 months without (even after cryopreservation ) alterations in their morphology, expression of stem cell markers, chondrogenic and myogenic differentiation potential. ${ }^{30}$ Gingival mesenchymal stem cells (GMSCs, isolated from gingival lamina propria) are a promising alternative to DMSCs because they are abundant, readily accessible and easily obtainable via minimal discomfort for the patient. The challenge is that GMSCs are usually highly heterogeneous cell population that can result in impaired self-renewal ability and multipotent differentiation capacity. The isolation of high purity GMSCs without fibroblast contamination is crucial for their successful application. Achieving this goal is not an easy task, because specific GMSC markers are not defined yet. On the other hand, in the course of cultivation, markers currently used in determination of the heterogeneous populations gradually decrease and disappear. ${ }^{31,32}$

\section{Concluding remarks}

Successful introduction of BTE in clinical practice requires overcoming of several challenges, including better clarification of biology and behavior of MSCs -one of the main players in this innovative therapeutic approach. We need to increase our knowledge about mechanisms of self-renewal and regulation of stem cell differentiation; to understand better the interactions between immune system and MSCs, to improve isolation, identification and cultivation methods, to learn more about specific characteristics of MSCs of various tissue origin and about the influence of individual patient/ donor features on the quality of the cells. Current and future joint efforts of biologists, chemists, engineers and clinicians focused on the design of optimized 3D scafolds; better study of the interactions between the scafold, the cells and the environment, and searching the ways of influencing them in the desired direction; establishing strategies to provide vascular support to the large osseous construct - all this will contribute to the advancement of regenerative medicine and its transformation into part of the routine orthopedic practice.

\section{Acknowledgements}

This study was supported by Grant № DFNI T 02 5/12.12.2014, Fund "Scientific Research", Bulgarian Ministry of Education and Science, and a bilateral project between Bulgarian Academy of Sciences (IEMPAM) and University of Technology in Kaunas, Lithuania.

\section{Conflict of interest}

The author declares no conflict of interest.

\section{References}

1. Akpancar S, Tatar O, Turgut H, et al. The current perspectives of stem cell therapy in orthopedic surgery. Arch Trauma Res. 2016;5(4):e37976.

2. Yousefi AM, James PF, Akbarzadeh R, et al. Prospect of stem cells in bone tissue engineering: a review. Stem Cells Int. 2016. 13 p.

3. Oryan A, Alidadi S, Moshiri A, Bone regenerative medicine: classic options, novel strategies, and future directions. J Orthop Surg Res. 2014;9(1):18.

4. Emara KM, Diab RA, Emara AK. Recent biological trends in management of fracture non-union. World J Orthop. 2015;6(8):623-628.
5. Roseti L, Parisi V, Petretta M, et al. Scaffolds for bone tissue engineering: state of the art and new perspectives. Mater Sci Eng C Mater Biol Appl. 2017;78:1246-1262.

6. Weissman IL. Stem cells: units of development, units of regeneration, and units in evolution. Cell. 2000;100:157-168.

7. Ramalho-Santos M, Willenbring H. On the origin of the term 'stem cell'. Cell Stem Cell. 2007;1(1):35-38.

8. ClinicalTrials.gov Bethesda, National Library of Medicine, MD US: Springer; 2000.

9. Komada Y, Yamane T, Kadota D, et al. Origins and properties of dental, thymic, and bone marrow mesenchymal cells and their stem cells. PLoS ONE. 2012;7(11):e46436.

10. Dominici M, Le Blanc K, Mueller I, et al. Minimal criteria for defining multipotent mesenchymal stromal cells. the international society for cellular therapy position statement. Cytotherapy. 2006;8(4):315-317.

11. Hilfiker A, Kasper C, Hass R, Mesenchymal stem cells and progenitor cells in connectivetissue engineering and regenerative medicine:is there a future for transplantation. Langenbecks Arch Surg. 2011;396(4):489 497.

12. Abumaree M, Al Jumah M, Pace RA, et al. Immunosuppressive properties of mesenchymal stem cells. Stem Cell Rev. 2012;8(2):375-392.

13. Friedenstein AJ, Gorskaja JF, Kulagina NN. Fibroblast precursors in normal and irradiated mouse hematopoietic organs. Exp Hematol.1976;4(5):267-274.

14. Bruder SP, Jaiswal N, Haynesworth SE. Growth kinetics, self-renewal, and the osteogenic potential of purified human mesenchymal stem cells during extensive subcultivation and following cryopreservation. $J$ Cell Biochem. 1997;64(2):278-294.

15. Bianco P, Riminucci M, Gronthos S, et al. Bone marrow stromal stem cells: nature, biology, and potential applications. Stem Cells. 2001;19(3):180-192.

16. Meza-Zepeda LA, Noer A, Dahl JA, et al. Highresolution analysis of genetic stability of human adipose tissue stem cells cultured to senescence. Journal of Cellular and Molecular Medicine. 2008;12(2):553-563.

17. Dahl JA, Duggal S, Coulston N, et al. Genetic and epigenetic instability of human bone marrow mesenchymal stem cells expanded in autologous serum or fetal bovine serum. The International Journal of Developmental Biology. 2008;52(8):1033-1042.

18. Follmar K, Decroos F, Prichard H, et al. Effects of glutamine, glucose, and oxygen concentration on the metabolism and proliferation of rabbit adipose-derived stem cells. Tissue Eng. 2006;12(12):3525-3533.

19. Ciuffi S, Zonefrati R, Brandi ML. Adipose stem cells for bone tissue repair. Clin Cases Miner Bone Metab. 2017;14(2):217-226.

20. Barlow S, Brooke G, Chatterjee K, et al. Comparison of human placenta- and bone marrow-derived multipotent mesenchymal stem cells. Stem Cells Dev. 2008;17(6):1095-1107.

21. Scherjon SA, De Groot-Swings GM, Claas FH, et al. Isolation of mesenchymal stem cells of fetal or maternal origin from human placenta. Stem Cells. 2004;22(7):1338-1345.

22. Brooke G, Tong H, Levesque JP, et al. Molecular trafficking mechanisms of multipotent mesenchymal stem cells derived from human bone marrow and placenta. Stem Cells Dev. 2008;17(5):929-940.

23. Hass R, Kasper C, Böhm S, et al. Different populations and sources of human mesenchymal stem cells (MSC): A comparison of adult and neonatal tissue-derived MSC. Cell Commun Signal. 2011;9:12. 
24. Darzi S, Werkmeister JA, Deane JA, et al. Identification and Characterization of Human Endometrial Mesenchymal Stem/Stromal Cells and Their Potential for Cellular Therapy. Stem Cells Transl Med. 2016;5(9):1127-1132.

25. Coutu DL, François M, Galipeau J. Mesenchymal stem cells and tissue repair. In: Allan D, Strunk D editors. Regenerative Therapy Using Blood-Derived Stem Cells. USA: Humana Press; 2012. p. 35-51.

26. Patel AN, Park E, Kuzman M, et al. Multipotent menstrual blood stroma stem cells: isolation, characterization, and differentiation. Cell Transplant. 2008;17(3):303-311.

27. Chalisserry EP, Nam SY, Park SH, et al. Therapeutic potential of denta stem cells. J Tissue Eng. 2017;8:2041731417702531.

28. D’Aquino R, Graziano A, Sampaolesi M, et al. Human postnatal dental pulp cells co-differentiate into osteoblasts and endotheliocytes: a pivotal synergy leading to adult bone tissue formation. Cell Death Differ. 2007;14(6):1162-1171.
29. Tatullo M, Marrelli M, Shakesheff KM, et al. Dental pulp stem cells: function, isolation and applications in regenerative medicine. $J$ Tissue Eng Regen Med. 2015;9(11):1205-1216.

30. Lizier NF, Kerkis A, Gomes CM, et al. Scaling-up of dental pulp stem cells isolated from multiple niches. PLoS ONE. 2012;7(6):e39885.

31. Du L, Yang P, Ge S. Isolation and characterization of human gingiva-derived mesenchymal stem cells using limiting dilution method. J Den Sci. 2016;11(3):304-314.

32. Górski B. Gingiva as a new and the most accessible source of mesenchymal stem cells from the oral cavity to be used in regenerative therapies. Postepy Hig Med Dosw. 2016;70(0):858-871. 